\title{
Protein kinase $\mathbf{G}$ phosphorylates soluble guanylyl cyclase and inhibits its activity
}

Andreas Papapetropoulos*1,3, Zongmin Zhou ${ }^{1}$, Nazish Sayed ${ }^{2}$, Anastasia Pyriochou ${ }^{3}$, Charis Roussos ${ }^{1}$, David Fulton ${ }^{4}$ and Annie Beuve ${ }^{2}$

\author{
Address: ${ }^{1}$ G.P. Livanos and M. Simou Laboratories, Evangelismos Hospital, Critical Care Department, University of Athens School of Medicine, \\ Athens, Greece, ${ }^{2}$ Department of Pharmacology, New Jersey Medical School, UDMNJ, Newark, NJ, USA, ${ }^{3}$ Laboratory of Molecular Pharmacology, \\ Department of Pharmacy, University of Patras, Patras, Greece and ${ }^{4}$ Vascular Biology Center, Medical College of Georgia, Augusta, GA, USA \\ Email: Andreas Papapetropoulos* - apapapet@upatras.gr \\ * Corresponding author
}

\author{
from $3^{\text {rd }}$ International Conference on cGMP Generators, Effectors and Therapeutic Implications \\ Dresden, Germany. 15-17 June 2007 \\ Published: 25 July 2007 \\ BMC Pharmacology 2007, 7(Suppl I):P45 doi:I0.1 186/I47I-2210-7-SI-P45
}

This abstract is available from: http://www.biomedcentral.com/I47I-22I0/7/SI/P45

(C) 2007 Papapetropoulos et al; licensee BioMed Central Ltd.

Soluble guanylyl cyclase (sGC) is a receptor for the signaling molecule nitric oxide (NO). Binding of $\mathrm{NO}$ to the sGC heme moiety causes up to 400-fold stimulation of its activity, leading to increased cGMP levels and cGMPdependent protein kinase (PKG) activation. As sGC subunits contain putative phosphorylation sites for PKG, we tested the hypothesis that sGC activity is regulated by PKG. In vitro kinase assays revealed that SGC is a PKG substrate. In vivo, a constitutively active form of PKG stimulated incorporation of ${ }^{32} \mathrm{P}$ into sGC. We then proceeded to map the exact phosphorylation site by generating serine to alanine mutations of putative PKG sites. Wild-type (wt) sGC co-expressed with a constitutively active form of PKG exhibited lower basal and NO-stimulated cGMP accumulation, while the serine to alanine $\mathrm{SGC}$ was resistant to the PKG-induced reduction in activity. In line with these observations, a phosphomimetic serine to aspartate sGC mutant showed reduced ability to synthesize cGMP. Using purified sGC and mutants, we observed that the phosphomimetic sGC mutant exhibited lower Vmax both under basal and NO-stimulated conditions and that the decrease in $\mathrm{Km}$ after NO stimulation was less pronounced than that for the wt. Moreover, the phosphorylation deficient sGC exhibited reduced desensitization to acute NO exposure and facilitated greater VASP phosphorylation. We conclude that PKG phosphorylates sGC on serine and phosphorylation inhibits sGC activity, establishing a negative feedback loop.

\section{Acknowledgements}

Supported by the Greek Ministry of Education and the Thorax Foundation. 